\title{
A counterpoint between computer simulations and biological experiments to train new members of a laboratory of physiological sciences
}

Marcelo Ozu, Ricardo A. Dorr, Facundo Gutiérrez, M. Teresa Politi and Roxana Toriano Advan in Physiol Edu 36:345-351, 2012.;

doi: 10.1152 /advan.00069.2012

You might find this additional info useful...

This article cites 24 articles, 6 of which you can access for free at:

http://advan.physiology.org/content/36/4/345.full\#ref-list-1

Updated information and services including high resolution figures, can be found at:

http://advan.physiology.org/content/36/4/345.full

Additional material and information about Advances in Physiology Education can be found at:

http://www.the-aps.org/publications/ajpadvan

This information is current as of December 3, 2012. 


\title{
A counterpoint between computer simulations and biological experiments
}

\section{to train new members of a laboratory of physiological sciences}

\author{
Marcelo Ozu, ${ }^{1}$ Ricardo A. Dorr, ${ }^{2}$ Facundo Gutiérrez, ${ }^{1}$ M. Teresa Politi, ${ }^{1}$ and Roxana Toriano ${ }^{1,2}$ \\ ${ }^{1}$ Laboratorio de Biomembranas, Departamento de Ciencias Fisiológicas, Facultad de Medicina, Universidad de Buenos \\ Aires, Buenos Aires, Argentina; and ${ }^{2}$ Consejo Nacional de Investigaciones Científicas y Técnicas, Argentina
}

Submitted 9 May 2012; accepted in final form 18 September 2012

Ozu M, Dorr RA, Gutiérrez F, Politi MT, Toriano R. A counterpoint between computer simulations and biological experiments to train new members of a laboratory of physiological sciences. Adv Physiol Educ 36: 345-351, 2012; doi:10.1152/advan.00069.2012.-When new members join a working group dedicated to scientific research, several changes occur in the group's dynamics. From a teaching point of view, a subsequent challenge is to develop innovative strategies to train new staff members in creative thinking, which is the most complex and abstract skill in the cognitive domain according to Bloom's revised taxonomy. In this sense, current technological and digital advances offer new possibilities in the field of education. Computer simulation and biological experiments can be used together as a combined tool for teaching and learning sometimes complex physiological and biophysical concepts. Moreover, creativity can be thought of as a social process that relies on interactions among staff members. In this regard, the acquisition of cognitive abilities coexists with the attainment of other skills from psychomotor and affective domains. Such dynamism in teaching and learning stimulates teamwork and encourages the integration of members of the working group. A practical example, based on the teaching of biophysical subjects such as osmosis, solute transport, and membrane permeability, which are crucial in understanding the physiological concept of homeostasis, is presented.

simulation; experimental; education; physiology; creativity

THE CREATIVE ACT is at the very top of the higher-order cognitive skills considered in the 2001 revision of Bloom's taxonomy of educational objectives (2). Creative thinking (which involves generating, planning, and producing, among others) is the most complex and abstract of all learning skills in the cognitive domain. An interesting analysis of the concept of creativity is to define it within two different theoretical frameworks: first, as a novel idea or solution to a problem that occurs in the mind of an individual and, second, as a social phenomenon that relies on the interaction among experts (10). The second framework is currently gaining importance, since there are data that show that new hypotheses or models are usually generated through discussions among knowledgeable peers (12). This social creativity thus relies strongly on the cognitive abilities of the members of the group.

When new members join a working group dedicated to scientific research, several changes occur in the group's dynamics. From a teaching point of view, a current challenge is to develop new learning strategies and tools that aim toward training new staff members in the process of creative thinking.

Address for reprint requests and other correspondence: R. Toriano, Laboratorio de Biomembranas, Departamento de Ciencias Fisiológicas, Facultad de Medicina, Universidad de Buenos Aires, Paraguay 2155, $7^{\circ}$ Piso, Ciudad Autónoma de Buenos Aires C1121ABG, Argentina (e-mail: rtoriano@fmed. uba.ar).
In this sense, bioinformatics, which is an unquestionably core component of present-day science, may also be considered crucial in the teaching of life sciences (8). In our days, there is a considerable growth in the use of educative software in all educational levels. This type of software, more or less participative, is generally used only as a demo of biological processes or as a knowledge test. We think bioinformatics can be used more profoundly as an educational tool.

Within the field of bioinformatics, computer simulation models are currently an essential tool in research in many scientific areas. According to Winslow (28), optimal modeling must satisfy certain requisites: 1 ) they should have a firm basis in experimental data; 2) they must make predictions that can be tested experimentally; and 3) finally, setting a superior goal, models should couple different levels of biological organization, an approach known as Noble's upward and downward pathways of causation (20). In addition, since models represent reality (19) but are not actually reality, one should understand what a model can and cannot explain (8). Such requisites are taken into account in our laboratory in the Department of Physiological Sciences of the School of Medicine of the University of Buenos Aires, where computational modeling and biological experiments are being used as tools to study water and solute transport across cellular membranes, with a particular emphasis on water movement observed with transmembrane osmotic gradients.

Osmosis and water transport. Several articles and reviews of research in transmembrane water transport have been published $(4,14,24,25)$. To facilitate the comprehension of this article, a brief reference framework is offered to the readers.

Osmosis is the net movement of water molecules (solvent) through a semipermeable membrane from a compartment with high water activity to a compartment with lower water activity. When teaching physiology, the concept of solute concentration is commonly used to refer to the phenomenon of osmosis as the flow of water molecules from the more dilute to the less dilute side (4). A semipermeable membrane is permeable to the solvent but not to the solute. In the absence of external hydrostatic pressure, the system reaches its equilibrium when solvent and solute concentrations on both sides are equal (Fig. 1A). In living cells, biological membranes can discriminate the passage of solutes; such membranes are called selective. The two main mechanisms by which water moves across the cellular membrane are simple diffusion across the phospholipid bilayer and, at a much higher rate, through water channels called aquaporins. Aquaporins are a family of small transmembrane proteins that are widespread in nature and can be found in all 
A

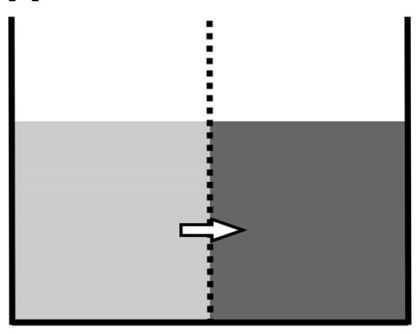

B

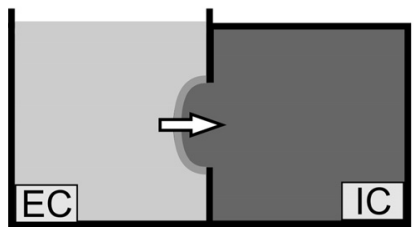

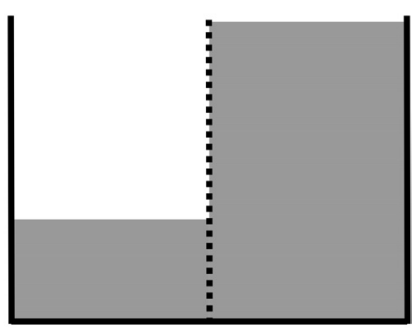

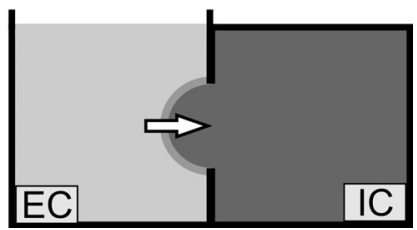

Fig. 1. Osmosis. A: diagram of osmosis. Left, a semipermeable membrane separates two compartments with anisotonic solutions. The osmotic gradient ( $\Delta \mathrm{Osm})$ is the difference in concentration between the two solutions on both sides of the membrane. Only water can get through the membrane (arrow). Right, passage of water through the membrane resulting in isotonic environments. The movement of water increases the volume of the solution in the compartment to the right. Dilution of the solution in this compartment occurs until an equilibrium is reached between the solutions in both compartments. At this moment, $\Delta$ Osm $=0$ and no net water flux occurs. The gray shading denotes the concentration of solutes in the medium. $B$ : diagram of osmosis in the experimental setup using emptied-out oocytes. Left, a cellular membrane separates two compartments with anisotonic solutions: the extracellular (EC) and intracellular (IC) compartments. The EC compartment is an open compartment; the IC compartment is a closed compartment. Water moves into the IC compartment driven by the osmotic gradient. Right, the entrance of water into the sealed compartment causes the displacement of the cellular membrane (cell swelling). The gray shading denotes the concentration of solutes in the medium. Parts are not to scale.

living Kingdoms (1). They initially were described as specific for water transport. ${ }^{1}$

Classically, aquaporin-mediated water transport was measured (using an osmotic swelling assay) in Xenopus laevis oocytes expressing heterologous aquaporins. This implies a two-compartment system (the inner side of the oocyte and the external medium) separated by a membrane (the oocyte cellular membrane). Oocyte swelling is driven by decreasing the osmolality of the medium bathing the extracellular side, which causes a hypotonic shock. Consequent changes in oocyte volume can be monitored by video microscopy $(11,29)$. The parameter used to characterize water movement through membranes is the osmotic permeability coefficient $\left(P_{\mathrm{f}}\right)(24)$. The volume change through time [defined as fluid flux $\left(J_{\mathrm{v}}\right)$ ] is related to $P_{\mathrm{f}}$ according to the following classical equation (29):

$$
J_{\mathrm{v}}(t)=P_{\mathrm{f}} A(t) \mathrm{V}_{\mathrm{w}} \Delta \operatorname{Osm}(t)
$$

where $A(t)$ is the area of the swelling cell and $\Delta \operatorname{Osm}(t)$ is the osmotic difference between the intracellular and extracellular media [both change through time $(t)$ ], and $\mathrm{V}_{\mathrm{w}}$ is the molar volume of water (which is assumed to be constant and equal to $18 \mathrm{ml} / \mathrm{mol}$ ). The amount of water molecules that cross the cellular membrane in each unit of time depends on the perme-

${ }^{1}$ Nowadays, some members of the aquaporin family are known to transport, at different rates, other substances, such as glycerol, urea, ammonium, metals, and gases.

ability of the membrane and the osmotic gradient between the intracellular and extracellular sides. In this article, we present experiments with human aquaporin 1 (hAQP1). AQP1 is a channel found in mammals that transports water at high rates. hAQP1 can be found in the red blood cells, kidney tubules, endothelium, heart, brain, and lungs, among other (5).

Educational approach. From an educational point of view, in this article, we share our group's experience of the arrival of new student members to the laboratory and their training in the field of water and solute transport through biological barriers. In silico modeling is presented as a tool that allows the teaching and learning complex physiological concepts and that complements the analysis and understanding of biological experimental results. We consider this a valuable experience that optimizes the acquirement of higher-order cognitive skills, such as creativity, in the frame of a social construction. We would like to point out that our work describes a learning intervention through an innovative use of basic technology. We believe that our practical experience can be extrapolated to other physiological fields.

\section{METHODS}

Here, we briefly describe the techniques applied in the biological and in silico experiments used throughout the educational experience.

Biological experiments. To obtain hAQP1 RNAs, plasmid construction and in vitro synthesis were taught and done as previously described by Ozu et al. (22). After the isolation of X. laevis oocytes, hAQP1 cRNA microinjection was done to express the protein in the cellular membrane (for details, see Ref. 22).

Experiments that evaluate cellular volume changes were done in emptied-out oocytes, as described in a previously published protocol (21). In brief, the membrane of an emptied-out oocyte was used as a diaphragm separating two compartments: a sealed chamber, representing the "intracellular" medium, and an open compartment with a volume 100 times larger, representing the extracellular medium. This allowed us to control the media composition on both sides of the membrane. After the extracellular medium was replaced with a hypotonic solution, cellular swelling occurred, induced by net water movement (Fig. 1B). This "inward" flux was estimated by video records of the cellular swelling. Relative volume changes were calculated as a function of time. The $P_{\mathrm{f}}$ value was obtained by fitting a nonlinear differential equation to the experimental data.

Animal study protocols were submitted to and approved by the Institutional Animal Care and Use Committee (Secretaría de Ciencia y Técnica, Facultad de Medicina, Universidad de Buenos Aires).

Mathematical modeling. Two computational models were used in this teaching and learning experience: the water movement model (WMM) and the solute and water movement model (SWMM). In the WMM, it is assumed that solutes cannot permeate the membrane and that only water can be transported through the membrane by osmosis. In this model, the system is characterized only by the $P_{\mathrm{f}}$ parameter. On the other hand, in the SWMM, solute transport through the oocyte membrane is also taken into account. Thus, in this model, the system is characterized by two parameters: $P_{\mathrm{f}}$ and the solute permeability coefficient $\left(P_{\mathrm{s}}\right)$.

Statistics. Data are presented as means \pm SE. $P$ values of $<0.05$ were considered statistically significant (by Student's $t$-test).

\section{RESULTS AND DISCUSSION}

Background. The new student members that join our staff are typically medical students who have strong theoretical knowledge in biology but little or no experience in working at the bench doing experiments. Another characteristic is that 
they have solid computer skills and are usually familiar at working with software as part of their extracurricular background.

When these staff members arrive at the laboratory, they must be trained in a first-approach theoretical introduction to basic (although sometimes complex) physiological and biophysical concepts, such as osmosis, diffusion, membrane transport, and membrane permeability. In parallel with this overview, they begin to perform experimental tasks with living cells, by manipulating $X$. laevis oocytes. Proper training is crucial to develop the necessary psychomotor skills to work with specific tools and instruments, especially in regard to performing biological experiments. For example, among the many psychomotor skills involved in our field, the ones that require special expertise are molecular biology techniques, the microinjection of cRNA, and the emptying out of oocytes (for technical details, see Refs. 21 and 22). At the same time, students are trained in the use of specific software to analyze images, graphically represent data, and perform statistical studies.

Recreating biological behavior. Traditionally, $P_{\mathrm{f}}$ is calculated by measuring changes in the volume of whole oocytes a few seconds after decreasing the osmolality of the extracellular medium $(11,29)$. This method has proved convenient for identifying aquaporins and for studying their biophysical properties (24). The emptied-out oocyte technique developed in our laboratory offers the advantage of being able to control both intracellular and extracellular medium compositions, which broadens experimental possibilities.

An example of experimental results obtained by the emptied-out oocyte approach is shown in Fig. $2 A$. Figure $2 A$ shows the time course of volume changes after a hypotonic shock. Each symbol is the mean of four independent experiments at a specific time. As shown in Fig. $2 A$, oocytes rapidly grew during the first few seconds. $P_{\mathrm{f}}$ was calculated from $E q .1$, where the osmotic gradient was imposed by changing the extracellular solution.

When the WMM (i.e., the mathematical model developed to simulate only the movement of water through the membrane) was tested, it showed a good adjustment with the experimental curve (Fig. 2A, solid line).

Thus, as a first conclusion, even very simple models can be sufficient to recreate and explain a biological behavior.

Prediction versus experimental data. Figure $2 A$ shows the best result of adjusting the WMM to the first $30 \mathrm{~s}$ of experimental data. From this adjustment, a simulated $P_{\mathrm{f}}$ value can be obtained. Such a value can be used to predict volume changes through time, in experiments that last $>30 \mathrm{~s}$. The emptied-out oocyte technique allows the performance of biological experiments in which volume can be measured for longer times after the hypotonic challenge (compared with the whole oocyte technique; Fig. 2B, solid squares). Therefore, predictions obtained from the WMM can be compared with experimental data. As shown in Fig. $2 B$, the predictive curve deviates from experimentally observed behavior. The WMM could be used to explain the behavior of the system up to $30 \mathrm{~s}$, but it is not enough to explain events that occur at longer times.

As a generalization, failures in models are highly informative, since they are a source of hypothesis generation. They stimulate further experimental testing and model refinement (28) and are thus useful for both students and scientists. For example, sometimes results show that a computer model must take into account additional biological variables to explain in vitro measurements.

Discriminating among computer models. When faced with clear differences between in silico predictions and biological experimental results, one may wonder about the biophysical causes behind those differences. This is the focus of the following experiments.

Since the oocyte cellular membrane is selective, it may be hypothesized that solute transmembrane movement also plays a role. A new model (SWMM) was thus designed to take solute movement into account. This model considers a membrane permeable to water and to an ideal unique solute. With a permeable solute, after a hypotonic shock, the total number of intracellular osmolytes varies with time. Considering that initial concentrations on both sides of the membrane are the same, one may expect intracellular osmolytes to exit the cell and extracellular water to enter the cell after the hypotonic shock, regaining osmotic equilibrium (Fig. 3A). Following this hypothesis, the interaction between both these movements could explain the flattening of the curve.

In Fig. 3B, the SWMM adjustment is shown along with experimental results. This curve had a good correlation with experimental data. One may think that the SWMM is "the best integral model," since it can explain experimental results both at short times (up to $30 \mathrm{~s}$ from the hypotonic shock) and in long-time protocols.

The results seem to suggest that a model with more parameters (in our example, $P_{\mathrm{f}}$ and $P_{\mathrm{s}}$ ) is better than a model with only one parameter (in this case, $P_{\mathrm{f}}$ ).
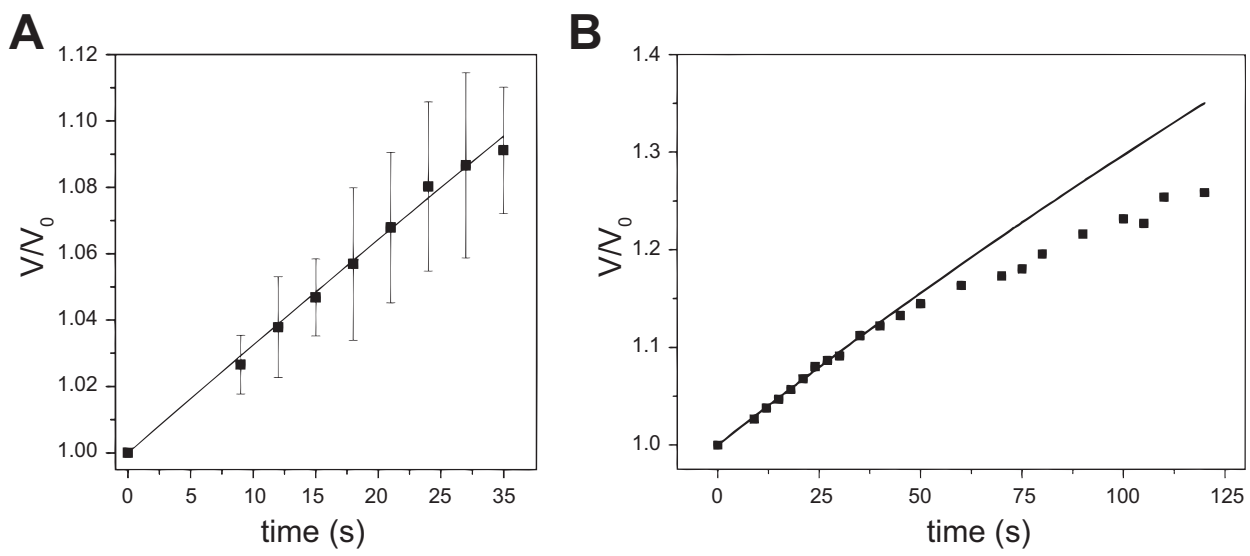

Fig. 2. Three learning steps: experimenting, evaluating, and predicting. $A$ : measurement of relative volume $\left(\mathrm{V} / \mathrm{V}_{0}\right)$ changes at short times. After a hypotonic shock by dilution of the external medium, volume changes were recorded by video microscopy in emptied-out oocytes expressing human aquaporin 1 . Solid squares represent biological experimental data; the solid line represents an adjustment considering the water movement model (WMM). $\Delta$ Osm $=150$ $\operatorname{mosM} ; n=4$. $B$ : measurement of $\mathrm{V} / \mathrm{V}_{0}$ changes at long times. Volume changes were measured at longer times, by applying a protocol similar to that shown in $A$. Solid squares represent biological experimental data; the solid line represents the prediction made by applying the WMM to a value of water permeability $\left(P_{\mathrm{f}}\right)$ calculated in the experiments shown in $A$. 

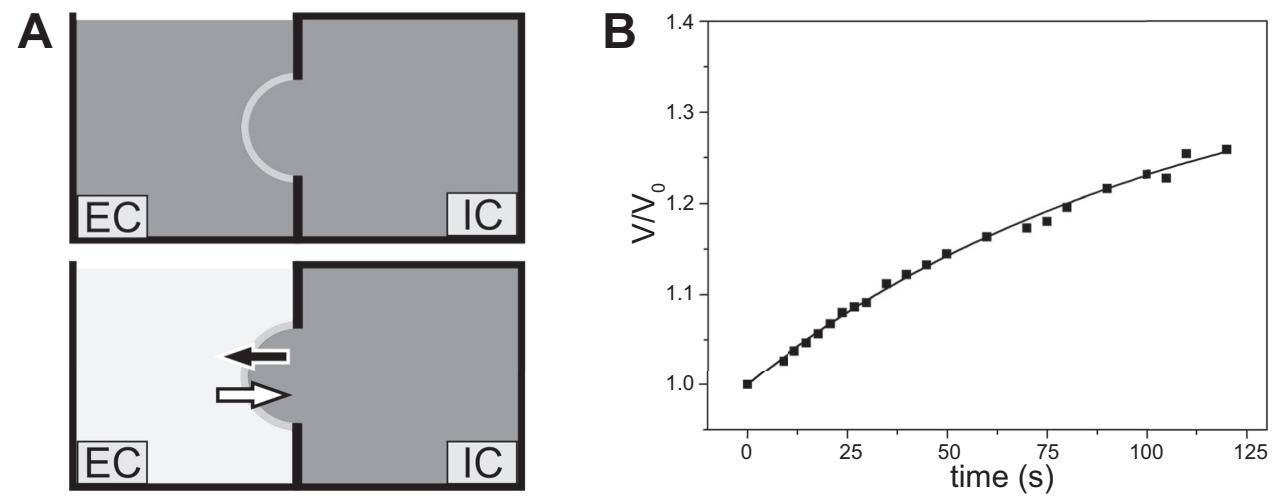

Fig. 3. Addition of variables to a computational model. A: diagram of the theoretical frame used to plan experiments. Top, initial condition. The same experimental media containing permeable solutes is found on the EC and IC sides of the emptied-out oocyte membrane. Bottom, after a hypotonic shock produced by diluting the external medium, there was a net movement of water (open arrow) from the EC compartment toward the IC compartment. Meanwhile, solutes (solid arrow) exit the IC compartment. The gray shading denotes the concentration of permeable solutes in the medium. $B$ : time course of $\mathrm{V} / \mathrm{V}_{0}$ changes in hyposmotic experiments with permeable solutes. Solid squares represent biological experimental data; the solid line represents the solute and water movement model (SWMM) adjustment. $\Delta$ Osm $=150$ mosM.

Evaluating the biological significance of a simulation. As Costa et al. (8) stated, researchers should always understand what a model can and cannot explain, which is also a key aspect of teaching and learning. In this sense, the results shown in Fig. $3 B$ indicate that the SWMM is correct from a mathematical point of view but does not consider whether or not the model makes biological sense.

To evaluate the biological significance of a simulation, a valuable tool is to compare it with experimental results or with well-known published data. In our case, even though the simulated $P_{\mathrm{f}}$ value is in accordance with published values (Table 1), some doubts appear in relation to $P_{\mathrm{s}}$ values. Experimental results published in previous literature have indicated that $P_{\mathrm{s}}$ values must be within the range of $1 \times 10^{-6}-1 \times 10^{-8}$ $\mathrm{cm} / \mathrm{s}(9,26)$. Although these published $P_{\mathrm{s}}$ values are indeed lower than those obtained by simulation with the SWMM $\left(P_{\mathrm{s}}=1.8 \times 10^{-5} \mathrm{~cm} / \mathrm{s}\right)$, they are not so far off as to exclude this model from being a biological possibility. Therefore, the SWMM could have biological sense.

To finally accept or discard this hypothesis, additional experiments were needed. With this in mind, the more experienced members of the group helped newcomers to design in vitro and in silico experiments, applying different strategies based on previous experimental reports (23). In this sense, a key experimental procedure to accept or discard the role of permeable solute movement as the cause of the flattening of the curve would be to plan experiments where solutes move in the same direction as water molecules do. From a theoretical point of view, these movements can be obtained by imposing an osmotic gradient with an impermeable solute but with identical initial concentrations of permeable solutes on both sides of the membrane (Fig. 4A). Thus, experiments using mannitol as the impermeable solute were planned.
Our biological results, shown in Fig. $4 B$, were sufficient to rule out the hypothesis that solute movement, opposite to the movement of water, is the main cause for volume growth to have a tendency to plateau. In this new experimental condition, even though water and permeable solutes move in the same direction, the tendency to plateau still remains (compare the results shown in Figs. $3 B$ and $4 B$ ). As a step further, new members also worked on reformulating the SWMM to consider the influence of a mannitol gradient. After the simulation was completed, there was no overlap between the simulated curve (Fig. 4B, solid line) and experimental data.

The discrepancies between in vitro and in silico results reinforce the idea that additional biological variables must be considered and integrated into a computer model.

Same results with different models. As described in Recreating biological behavior in RESULTS AND DISCUSSION, the WMM adjusts very well to experimental data up to $30 \mathrm{~s}$. Nevertheless, considering that the oocyte cellular membrane is selective, the participation of solute movement during the first seconds of the experiment must not be discarded. When tested, the SWMM had an adjusted result that was similar to the WMM. Indeed, $P_{\mathrm{s}}$ calculated with the SWMM at short times was very low $\left(P_{\mathrm{s}}<\right.$ $\left.1 \times 10^{-10} \mathrm{~cm} / \mathrm{s}\right)$ and had no significant difference with zero. Thus, the SWMM reduces itself to the WMM when applied to the initial seconds. Since hAQP1 transports water at high rates (a discussion about this topic can be found in Ref. 27), it seems reasonable to use the WMM during the first few seconds of the hypotonic response. It also justifies why, in classical whole oocyte experiments published in previous literature, measurements done during the first seconds after a hypotonic challenge are accepted as representative to calculate $P_{\mathrm{f}}$.

As a new conclusion, it is interesting to show new group members how different models can produce similar results.

Table 1. Evaluating the biological significance of a computational adjustment

\begin{tabular}{lcccc}
\hline \hline & Biological Experiment & Water Movement Model & Solute and Water Movement Model & Published Values \\
\hline Permeability coefficient, $\mathrm{cm} / \mathrm{s}$ & $3 \times 10^{-3}$ & $2.5 \times 10^{-3}$ & $3.4 \times 10^{-3}$ & $1.72 \times 10^{-3 *}$ \\
Solute permeability coefficient, $\mathrm{cm} / \mathrm{s}$ & & & $1.8 \times 10^{-5}$ & $1 \times 10^{-6} \dagger$ to $1 \times 10^{-8}$ † \\
\hline
\end{tabular}

*Osmotic permeability coefficient value in oocytes expressing human aquaporin 1 (22). $\dagger$ Direct measurements of sodium by tracer-flux analysis in the presence of calcium (26). $\ddagger$ Values for the sodium permeability coefficient under voltage clamp (9). 


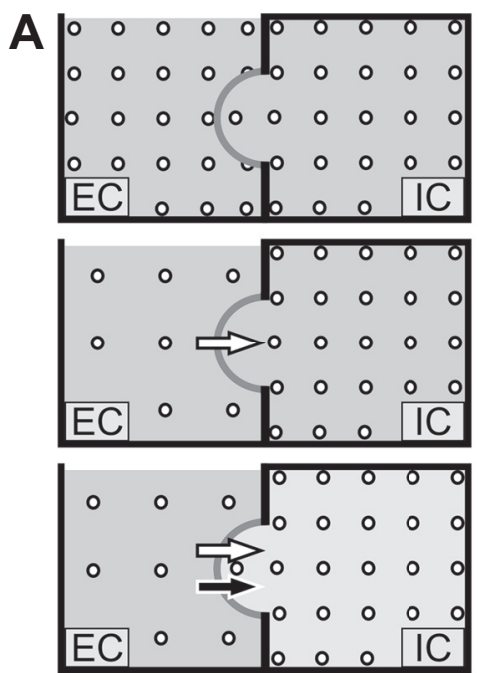

B

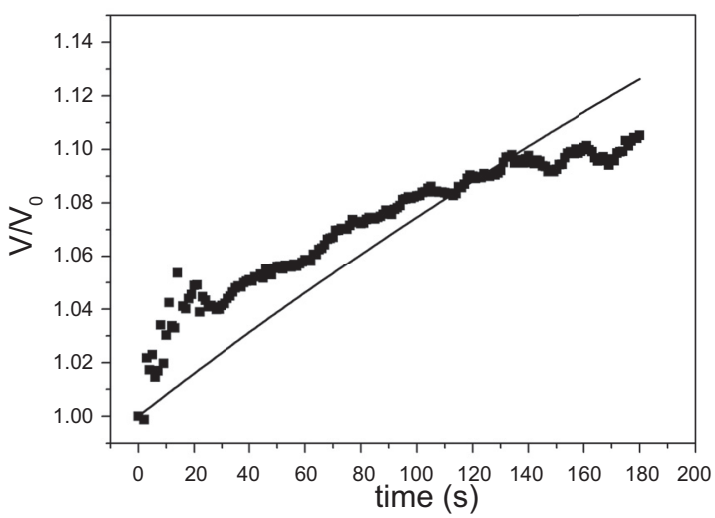

Fig. 4. Planning new strategies. A: diagram of the theoretical frame used to plan new experiments. Top, initial condition. The composition of the solutions on both sides of the membrane was the same. The gray shading denotes the concentration of permeable solutes in the medium. Open circles represent impermeable solute molecules. Middle, a hypotonic shock was applied by replacing the external medium with another solution that had a lower concentration of impermeable solutes but maintained the same concentration of permeable solutes. The open arrow represents the net water movement from the EC compartment to the IC compartment. Bottom, as a consequence of this net water movement, the concentration of permeable solutes in the IC medium decreased (notice the lighter shade of gray). This created a concentration gradient for permeable solutes, which moved through the selective membrane (solid arrow) in the same direction as water did (open arrow). B: time course of $\mathrm{V} / \mathrm{V}_{0}$ changes after a hypotonic shock imposed by a gradient of mannitol in the presence of a permeable solute. The composition of initial EC and IC solutions was mannitol $(0.2 \mathrm{~mol} / \mathrm{l})$ plus permeable solutes $(0.16 \mathrm{~mol} / \mathrm{l})$. A hypotonic shock was imposed by changing the EC medium ( $0.05 \mathrm{~mol} / 1 \mathrm{mannitol}$ plus $0.16 \mathrm{~mol} / \mathrm{l}$ permeable solutes). Solid squares represent biological experimental data; the solid line represents the simulated prediction made with a reformulated SWMM.

When faced with such a situation, the choice of one model over another must prioritize simplicity.

In our example, the SWMM is more complex than WMM and thus implies an additional "cost" of information, which reduces the degrees of freedom of the system. In this case, an algorithm with more parameters is not necessary for short-time experiments, making the WMM the model of choice for calculating $P_{\mathrm{f}}$.

General aspects and conclusions. New members working in a scientific group modify its dynamics. If new entrants are students or young professionals who just graduated, trained researchers spend part of their time in guiding these new members in the acquisition and understanding of different experimental methodologies in the analysis of data and in the formulation of hypotheses. In summary, part of the training that new members receive aims toward the acquisition of skills for making independent decisions.

We propose to emphasize the teaching of methods that involve a counterpoint between computer simulation and biological experiments as a tool for both teaching and learning physiology. This learning methodology allows newcomers to test and make use of essential laboratory tools even very early in their research experience. It also fosters a way of thinking and working according to the scientific method.

We conclude that computer simulation does not replace, although it does complement and enrich, the use of classic experimental methods. We consider that the analysis and development of algorithms used in computer simulations promote in new scientists a mathematical approach toward understanding physiological phenomena. The possibility of easily modifying experimental variables by simulation widely broadens the range of phenomena that can be studied. The analysis of predictions made with in silico techniques, contrasted with biological experiments, offers a more thorough comprehension of the biophysical processes involved and helps develop new hypotheses. Simulations need to find justification in experiments, and experiments need a model, based on theory and simulation (3). In this sense, our own experience coincides with Winslow's statement: discrepancies between model predictions and experimental outcomes may suggest that additional biological processes must be added to a model or that some existing component of the model needs a more precise characterization (28).

A recent update of Bloom's revised taxonomy has included in the learning domains new behaviors and actions emerging from technology and digital advances (7). Concomitantly with the cognitive descriptors mentioned in this article (such as "using," "manipulating," "comparing," "testing," "hypothesizing," "experimenting," or "planning"), new verbs [such as "googling," "searching" (as applied to the field of bibliographic research), "refactoring" and "programming" (with the latter in the creative category)] are starting to be used. All of them describe digital-related actions that take part in the education and training of new staff members.

The cognitive and psychomotor skills in Bloom's learning domains have been mentioned along the description of our particular experience. Additionally, we also emphasize the need to reinforce skills in the affective domain: personalized instruction, tutorial modes, and the interrelation among old and new members especially stimulate group bonding and teamwork.

We think that the recreation of biological behaviors, the validation of prediction, the discrimination among computer models, and the evaluation of the biological significance of a simulation are universal steps in the counterpoint between computer simulation and biological experiments approaches 
with educational (and scientific) purposes. In conclusion, we believe that our particular experience can serve to inspire educators from other areas to seek new teaching strategies in the training of young researchers who are new to laboratory work.

\section{APPENDIX: COMPUTATIONAL MODELS}

Here, we provide the details of the computational models used in this work, for those who want to apply the described methodology to the study of water and solute transport. The authors also make available the software used to those who request it.

Both of the models here described are adapted from the twoparameter transport formalism used by Jacobs in 1933 (16), as reviewed by Kedem and Katchalsky in 1958 (17) and by Kleinhans in 1998 (18). The SWMM is also an adaptation of the model by Hernandez and Cristina (15).

The WMM. Simulations were performed to study the osmotic response of $X$. laevis oocytes expressing hAQP1. The model used was based on the general formulation used for fluid fluxes (13). The following assumptions were taken into account:

1. Hypotonic shock was modeled using a step function (Heviside function). Then, it was assumed that at $t=0 \mathrm{~s}$, extracellular concentration was equal to intracellular concentration. Suddenly, the extracellular concentration was instantly lowered.

2. A semispherical shape was assumed for the intracellular compartment.

3. The oocyte membrane was assumed to be permeable to water.

4. There were no interactions between membrane electrical potential and water flux.

5. The membrane area involved in water flux was considered to be variable and dependent of intracellular volume, as follows:

$$
A(t)=\frac{1}{2} 4 \pi r^{2} 9
$$

where $r$ is the radius of the oocyte and the value of 9 corrects the observed area by taking into account the invaginations of the membrane (6).

6. Water could be transported by an osmotic mechanism. The following phenomenological expression is proposed:

$$
J_{\mathrm{v}}(t)=P_{\mathrm{f}} A(t) \mathrm{V}_{\mathrm{w}} 10^{-3}\left[\frac{m_{\mathrm{i}}}{\mathrm{V}_{\mathrm{i}}(t)}-C_{\mathrm{e}}\right]
$$

where $\mathrm{V}_{\mathrm{W}}$ is the water molar partial volume, $m_{\mathrm{i}}$ is the total number of osmolytes, $\mathrm{V}_{\mathrm{i}}(t)$ is the internal volume, and $C_{\mathrm{e}}$ is the extracellular osmolarity.

The term

$$
\left[\frac{m_{\mathrm{i}}}{\mathrm{V}_{\mathrm{i}}(t)}-C_{\mathrm{e}}\right]
$$

represents the osmotic force.

7. The rate of internal volume was only affected by the water flux (continuity theorem), as follows:

$$
\mathrm{dV}_{\mathrm{i}}(t) / \mathrm{d} t=J_{\mathrm{v}}(t)
$$

The total solute concentration was computed in terms of $m_{\mathrm{i}}(t)$ and $\mathrm{V}_{\mathrm{i}}(t)$, as follows:

$$
C_{\mathrm{i}}(t)=m_{\mathrm{i}}(t) / \mathrm{V}_{\mathrm{i}}(t)
$$

$\mathrm{V}_{\mathrm{i}}$ was calculated from the image of the emptied-out oocyte, assuming a semispherical shape.

8. The membrane was assumed to be impermeable to solutes. Thus, the total number of intracellular osmolytes was constant.
The SWMM. In the case of the SWMM, all the previous assumptions from the WMM were valid except for assumption 8, which was replaced by $8^{\prime}$ :

$8^{\prime}$. The membrane was assumed to be permeable to water and to an ideal unique solute. The total number of intracellular osmolytes was variable and was updated with each iteration step. There were no interactions between membrane electrical potential and solute flux.

The addition of the following equation modified the WMM to take into account solute flux:

$$
J_{\mathrm{s}}(t)=-P_{\mathrm{s}} A(t) 10^{-3}\left[\frac{m_{\mathrm{i}}(t)}{\mathrm{V}_{\mathrm{i}}(t)}-C_{\mathrm{e}}\right]
$$

where $J_{\mathrm{s}}(t)$ is the mean solute flux across the membrane $\left[\mathrm{d} m_{\mathrm{i}}(t) / \mathrm{d} t=J_{\mathrm{s}}(t)\right]$ and $P_{\mathrm{s}}$ is defined as the solute permeability.

9. In the case of imposing a hypotonic shock with mannitol, the water flux value was calculated considering the movement of permeable solutes and the presence of mannitol. The permeable solute mass was updated using the following equation: $\mathrm{d} m / \mathrm{d} t=A(t) P_{\mathrm{s}}\left[C_{\mathrm{es}}-\right.$ $\left.\left(m_{\mathrm{is}} / \mathrm{V}_{\mathrm{i}}\right)\right]$, where $m_{\mathrm{is}}$ is the intracellular permeable solute mass and $C_{\mathrm{es}}$ is the extracellular permeable solute concentration.

Simulations. Simulations were performed using the Euler method with custom-made software developed with Visual Basic code, using an integration step of $1 \mathrm{~s}$. This step guaranties the order of accuracy and the stability of the Euler method. The procedure predicts the time course of relative volume changes that occur after a hypotonic challenge.

\section{ACKNOWLEDGMENTS}

The authors thank to Dr. Osvaldo Chara, who wrote the backbone code of the WMM and SWMM algorithms.

\section{GRANTS}

This work was supported by Agencia Nacional de Promoción Científica y Tecnológica Grant ANPCyT-PICT 32988, Consejo Nacional de Investigaciones Científicas y Técnicas Grant CONICET-PIP 5926, and Universidad de Buenos Aires Grant UBACyT M824.

\section{DISCLOSURES}

No conflicts of interest, financial or otherwise, are declared by the author(s).

\section{AUTHOR CONTRIBUTIONS}

Author contributions: M.O., R.A.D., and R.T. conception and design of research; M.O., F.G., and M.T.P. performed experiments; M.O., R.A.D., F.G., M.T.P., and R.T. analyzed data; M.O., R.A.D., F.G., M.T.P., and R.T. interpreted results of experiments; M.O., R.A.D., F.G., M.T.P., and R.T. prepared figures; M.O., R.A.D., F.G., M.T.P., and R.T. drafted manuscript; M.O., R.A.D., F.G., M.T.P., and R.T. edited and revised manuscript; M.O., R.A.D., F.G., M.T.P., and R.T. approved final version of manuscript.

\section{REFERENCES}

1. Agre P, King LS, Yasui M, Guggino WB, Ottersen OP, Fujiyoshi Y, Engel A, Nielsen S. Aquaporin water channels-from atomic structure to clinical medicine. J Physiol 542: 3-16, 2002.

2. Anderson L, Krathwohl DA. Taxonomy for Learning, Teaching and Assessing: a Revision of Bloom's Taxonomy of Educational Objectives. New York: Longman, 2001.

3. Beckstein O. Teaching old coefficients new tricks: new insight into the meaning of the osmotic and diffusive permeation coefficients. Biophys $J$ 96: 763-764, 2009.

4. Ben-Sasson SA, Grover NB. Osmosis: a macroscopic phenomenon, a microscopic view. Adv Physiol Educ 27: 245, 2007.

5. Benga G. The first discovered water channel protein, later called aquaporin 1: molecular characteristics, functions and medical implications. Mol Aspects Med 33: 518-534, 2012.

6. Chandy G, Zampighi GA, Kreman M, Hall JE. Comparison of the water transporting properties of MIP and AQP1. J Membr Biol 159: 29-39, 1997. 
7. Churches A. Bloom's Digital Taxonomy (online). http://edorigami.wikispaces. com/Bloom's+Digital+Taxonomy [26 September 2012].

8. Costa MJ, Galembeck E, Marson GA, Torres BB. A quick guide for computer-assisted instruction in computational biology and bioinformatics. PLoS Comput Biol 4: e1000035, 2008.

9. Costa PF, Emilio MG, Fernandes PL, Ferreira HG, Ferreira KG. Determination of ionic permeability coefficients of the plasmamembrane of Xenopus laevis oocytes under voltage clamp. J Physiol 413: 199-211, 1989.

10. DeHaan RL. Science education. Teaching creative science thinking. Science 16: 1499-500, 2011.

11. Dorr R, Ozu M, Parisi M. Simple and inexpensive hardware and software method to measure volume changes in Xenopus oocytes expressing aquaporins. J Neurosci Methods 161: 301-305, 2007.

12. Dunbar K. How scientists really reason: scientific reasoning in real-world laboratories. In: Mechanisms of Insight, edited by Sternberg RJ, Davidson J. Cambridge, MA: MIT Press, 1995.

13. Finkelstein A. Water Movement Through Lipid Bilayers, Pores, and Plasma Membranes. New York: Wiley \& Sons, 1987.

14. Goodman BE. Transport of small molecules across cell membranes: water channels and urea transporters. Adv Physiol Educ 26: 146-157, 2002.

15. Hernández JA, Cristina E. Modeling cell volume regulation in nonexcitable cells: the roles of the $\mathrm{Na}^{+}$pump and of cotransport systems. Am J Physiol Cell Physiol 275: C1067-C1080, 1998.

16. Jacobs MH. The simultaneous measurement of cell permeability to water and to dissolved substances. J Cell Comp Physiol 2: 427-444, 1933.

17. Kedem O, Katchalsky A. Thermodynamic analysis of the permeability of biological membranes to non-electrolytes. Biochim Biophys Acta 27: 229-246, 1958.

18. Kleinhans FW. Membrane permeability modeling: Kedem-Katchalsky vs. a two-parameter formalism. Cryobiology 37: 271-289, 1998.
19. Kohl P, Hunter PJ, Winslow RL. Model interactions: "it is the simple, which is so difficult". Prog Biophys Mol Biol 107: 1-3, 2011.16.

20. Noble D. Biophysics and systems biology. Phil Trans $R$ Soc A 368: 1125-1139, 2010.

21. Ozu M, Dorr R, Parisi M. New method to measure water permeability in emptied-out Xenopus oocytes controlling conditions on both sides of the membrane. J Biochem Biophys Methods 63: 187-200, 2005.

22. Ozu M, Dorr RA, Politi MT, Parisi M, Toriano R. Water flux through human aquaporin 1: inhibition by intracellular furosemide and maximal response with high osmotic gradients. Eur Biophys $J$ 40: 737-746, 2011.

23. Ozu M, Toriano R, Capurro C, Parisi M. Electrical parameters and water permeability properties of monolayers formed by T84 cells cultured on permeable supports. Braz J Med Biol Res 8: 133-140, 2005.

24. Parisi M, Dorr RA, Ozu M, Toriano R. From membrane pores to aquaporins: 50 years measuring water fluxes. J Biol Phys 33: 331-343, 2008.

25. Reuss L. Water Transport Across Cell Membranes (online). doi:10.1002/ 9780470015902.a0020621.pub2 [26 September 2012].

26. Tupper J, Maloff B. The ionic permeability of the amphibian oocyte in the presence or absence of external calcium. J Exp Zool 185: 133-144, 1973.

27. Wan X, Steudle E, Hartung W. Gating of water channels (aquaporins) in cortical cells of young corn roots by mechanical stimuli (pressure pulses): effects of ABA and of $\mathrm{HgCl}_{2} . J$ Exp Botany 55: 411-422, 2004.

28. Winslow RL. Grand challenges in computational physiology and medicine. Front Physiol 2: 79, 2011.

29. Zhang R, Logee K, Verkman AS. Expression of mRNA coding for kidney and red cell water channels in Xenopus oocytes. J Biol Chem 265: 15375-15378, 1990.

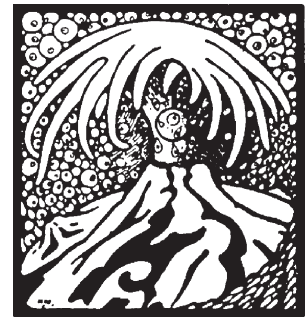

